\title{
Epidemiology of stroke in the elderly in the Nordic countries. Incidence, survival, prevalence and risk factors
}

\author{
Torgeir Engstad ${ }^{1}$, Tora Torgersen Engstad ${ }^{2}$, Matti Viitanen ${ }^{3}$ and Hanne Ellekjær ${ }^{4}$ \\ 1) Department of Geriatrics, University Hospital of Northern Norway, Tromsø, Norway \\ 2) Department of Geriatrics, Diakonhjemmet Hospital, Oslo, Norway \\ 3) Department of Geriatrics, University of Turku, Finland \\ 4) The Stroke Unit, Department of Internal Medicine, St Olavs Hospital, Trondheim, Norway \\ E-mail: torgeir.engstad@me.com Telephone:+4790143832
}

\begin{abstract}
Objective: To review what is known at present with respect to incidence, survival, risk factors and prevalence among the elderly stroke patients in the Nordic countries.

Method: This article is based mainly on literature identified through search engines (Mc Master Plus, Cochrane Library, Medline and PubMed), restricted to first-ever stroke in Nordic population-based studies and having applied to the standard WHO definition, a prospective study design and no upper age limit.

Results: Data from the Nordic countries show an incidence rate of 1250 to $1796 / 100000$ in the age group $75-84$, and 1628 to 2234 in those above 85 years. The incidence rates are higher among men, but women are expected to contribute more to incident cases due to their higher life expectancy. If the age-specific incidence of stroke remains stable, the proportion of stroke patients aged 80 years and older may reach $50 \%$ in a few decades. The elderly stroke patients have a higher 30-days case fatality, and a higher risk of dependency. Better treatment of stroke patients has improved the survival over the last two decades. The prevalence is expected to increase due to the decrease in lethality, a slower fall in incidence and a higher proportion of elderly. Cardiovascular risk factors increase with age. Hypertension is a major risk factor for stroke mortality in the elderly. Cardioembolic stroke due to atrial fibrillation is the most common stroke subtype in the elderly. Lifestyle risk factors are less prevalent in the older stroke patients.

Conclusion: The growing proportion of elderly stroke patients is a major challenge for future stroke care. The elderly stroke patients have a different risk factor profile compared to younger stroke patients. Treatment should focus on regaining independency. The age-specific epidemiology of stroke needs to be studied further in large studies in order to plan for future health care.
\end{abstract}

\section{INTRODUCTION}

The elderly stroke patients are likely to become one of the fastest growing patient groups in the years to come due to future demographic changes with a higher proportion of elderly in the population. The proportion of elderly 80 years and older in Norway and other Nordic countries will increase from $4-5 \%$ of the population to $8 \%$ in 2040 (Nordic Statistical yearbook 2010). The life expectancy at birth in Norway in 2009 was 78.6 years for men and 83.1 years for women.

Today, more than $50 \%$ of all strokes occur in those over the age of 75 , and as much as $30 \%$ among those over 85. For a person suffering a stroke, the consequences are often functional disabilities and poor lifesatisfaction, with a corresponding burden for carers and family. This is especially true for the more fragile, elderly stroke patients.

In order to plan for the necessary level of health care, and to implement preventive measures, knowledge of stroke epidemiology of the elderly and their exposure to modifiable risk factors are needed (1). Unfortunately, the very elderly are excluded from most clinical stroke trials (2).

This paper is an attempt to review what is known at present with respect to incidence, survival, risk factors and prevalence among the elderly stroke patients in the Nordic countries.

\section{METHODS}

Studies on stroke epidemiology of the elderly were identified through the search engines Mc Master Plus, Cochrane Library, Medline and PubMed. The search is restricted to first-ever stroke (3) in Nordic populationbased studies after the 1980s. Eligibility criteria were stroke according to the standard WHO definition, studies with prospective study design and without any upper age limit (4).

Some studies from European countries other than the Nordic are commented on to present the current knowledge of stroke epidemiology of the elderly.

Trends in mortality are based on the Norwegian Cause of Death register, Statistics Norway.

\section{RESUlTS}

\section{Incidence}

The mean age at onset of a first-ever stroke in Norway is 75 years for men and 78 for women (5). The incidence of stroke increases steeply with age. 
Recurrent stroke contributes to nearly $25 \%$ of all incident strokes, $23 \%$ in the Copenhagen Stroke Study, and $27 \%$ in Innherred, Nord-Trøndelag, respectively.

A review of stroke incidences in Europe by Truelsen et al. (6) includes age and sex specific incidence rates for the Nordic countries. For the age group 75-84 the male incidence rates (per 100000 ) vary between 1301 (Iceland) and 1796 (Norway), and the female rates between 1250 (Denmark) and 1493 (Iceland). The corresponding incidence rates for the age group above 85 vary between 1697 (Iceland) and 2234 (Norway) for men, and 1628 (Denmark) and 1990 (Iceland) for women.

There is a tendency of a higher incidence in men as compared to women. Among the Nordic countries Iceland is the only exception reporting a higher incidence in women than men. Women are, as a consequence of their higher life expectancy, predicted to contribute more to incident cases of stroke over the age of 85 years in absolute numbers, as the population is shifted towards a higher age.

In a hospital based registry from Bergen, an incidence rate of 880 per 100000 in the age group 80-90 years and 1612 per 100000 in age group 90 years or older were reported for ischemic stroke (7). In Finland the national stroke database registered 104899 firstever stroke patients, median age 74 years, over the years 1999-2008. During this period the stroke incidence rates decreased for the age groups 75-84, 85-94 and 95+ with 18, 15 and 3 percent, respectively (8). This decrease in the Finnish stroke incidence seems to be in line with a worldwide 42 percent decrease in all stroke incidence over the last 4 decades, restricted to highincome countries (4).

In Norway time trend incidence studies are lacking, but studies from Sweden and Denmark do not convincingly support a decline in incidence.

If the age-specific incidence of stroke remains stable, the proportion of elderly stroke patients will increase substantially in the forthcoming decades (9-11). Data collected after the year 2000 from 5 European countries and the United States, report similar trends of age and sex-specific incidences of stroke in a recent review by Zhang et al. (12) A study from Lund (10) predicts that the proportion of stroke patients aged 80 years and older will increase from $37 \%$ in 2005 to $50 \%$ in 2050 , if the age-specific incidence rates remain stable.

\section{Survival and functional outcome}

Data from several European countries indicate that between one-third to half of all persons with stroke die from stroke, of which the estimated 28-days case fatality rate is $20 \%$ (6). A population based study from Nord-Trøndelag 1994-96 reported an overall 30-days case fatality of $19 \%$ for first-ever stroke, $22 \%$ and $25 \%$ in the age group 75-84 and 85 years and above, respectively (13).

Data from Lund in 2001-2002 and Ørebro 19992001 reported a total 28 -days fatality of $14.3 \%$ and $19 \%$ for the same age groups (14). In 1997 FINSTROKE reported a 28 -days case-fatality rate for all stroke patients of $28.2 \%$ (8).

The 28-30 days case fatality mainly depends on age, stroke severity and stroke subtype, whereas the late case fatality is caused by cardiovascular risk factors such as atrial fibrillation, smoking, diabetes mellitus and ischemic heart disease.

Stroke related lethality is consistently higher among the elderly, and increases steeply with age. According to Russo the elderly have a higher 30-days case fatality rate as compared to those younger (OR 3.07) (15).

For ischemic stroke the 28-days case fatality in Finland decreased from $13 \%$ in 1999 to $10 \%$ in 2000 , whereas the corresponding percentages for intracerebral hemorrhage was 32.2 and 29.3, respectively (8).

A 30-years follow-up of the Copenhagen Stroke Study, reported the long-term survival for men steadily improving from 1978 to 2001, with a similar pattern for women.

In general, long-term survival from stroke seems to be improving more than short-term survival. The increased long-term survival contributes to a higher prevalence, as stroke is becoming more of a chronic illness.

Half of those surviving a stroke, fail to regain independency assessed by Modified Rankin Scale $>2$ three months after stroke (Bent Indredavik, personal communication, Stroke register, St Olavs hospital). Elderly stroke survivors have a higher risk of dependency than younger stroke patients, with an odds ratio of 1.77 in a study of elderly above 80 years as compared to those younger than 80 (16).

\section{Mortality}

In 2010, the total number of stroke related deaths (ICD 10: I 60-69) was 3180 in Norway. Of these, a total of $2864(90 \%)$ were above the age of $70(17)$. The mortality of stroke has been steadily decreasing since the 1950-60s. The decrease in age specific mortality rate for men older than 70 years has been ongoing since 1970, and for women since 1965 .

The reason for declining mortality is not well understood; how much is due to a decreased incidence and how much is due to better survival after stroke?

As already mentioned, time trend incidence studies do not convincingly support a decline, although favorable trends in traditional and socioeconomic risk factors and use of prophylactic medication have been observed. Changes in diagnostic criterias and hospitalization rate may also have influenced the incidence reported. Observational studies have documented that better treatment of stroke patients has improved the survival over the last two decades (18).

\section{Prevalence}

In the Kungsholmen study, Sweden, the prevalence of stroke increased with age; from 9.7\% (95\% CI 5.4$13.9)$ in the age group $75-79$ years to $14.3 \%$ (95\% CI 
7.4-21.2) in those 85 years and older (19). In a study from Umeå, the prevalence of stroke in the groups 85$89,90-94$ and $\geq 95$ years was $21 \%$ in all groups (20). In Finland the prevalence in Vantaa in the age group 85 years and older was 20.1 (21).

The prevalence is expected to increase over the next couple of decades as a consequence of a sharper decrease in lethality than the predicted fall in incidence. The higher number of elderly will contribute to this trend, and to the total burden of vascular diseases in general.

\section{Risk factors and treatment effects}

The main modifiable risk factors for stroke are hypertension, coronary heart disease, atrial fibrillation, valvular pathology, hyperlipidaemia, diabetes, smoking, inactivity, and alcohol consumption. Cardiovascular diseases causing a higher risk of stroke increase dramatically with age.

In Vantaa hypertension was found in $30.6 \%$, diabetes in $22.5 \%$, heart failure in $68.5 \%$, atrial fibrillation in $35.1 \%$ and myocardial infarct in $17.1 \%$ of stroke patients 85 years and older.

Several studies show that lifestyle risk factors such as smoking, alcohol and obesity are important risk factors in younger stroke patients $<60$, but are less prevalent in the older stroke patients. A nationwide Danish registry showed that after the age 70-80 the prevalence of hypertension, diabetes mellitus, coronary heart disease, and for men atrial fibrillation, decrease (22).

In the very old stroke patients the frequencies of hypertension and atrial fibrillation are higher than in younger age groups. An Italian study including 535 stroke patients observed that hypertension and atrial fibrillation were more common in those over 80, whereas life style risks such as diabetes, hyperlipidemia, smoking and obesity were more frequent in the group younger than 80 years (23).

\section{Hypertension}

Hypertension is the most prevalent risk factor among the elderly and therefore the most important modifiable risk factor for stroke.

Based on health service data from the counties of Oslo, Bergen, Hedmark, Oppland, Trøndelag, Troms and Finnmark, there has been a steady decrease in hypertension in Norway over the last 20 years. A blood pressure study from Bergen with a 20 -year follow-up has demonstrated that the absolute increase in mortality with increasing blood pressure was greatest in persons aged 60-69 and 70-79. In Nord-Trøndelag, the proportion of participants with a blood pressure below 140/90 mmHg increased significantly between HUNT-2 and HUNT-3, and more so among the elderly.

Antihypertensive treatment is beneficial in those over 80 according to data from the recently published Hypertension in the Very Elderly Trial (24). A reduction of baseline systolic and diastolic blood pressure of $17 / 7 \mathrm{mmHg}$ and isolated systolic $\mathrm{BP}$ of $19.3 \mathrm{mmHg}$ as compared to the control group during a 2-year followup reduced the relative risk of having a stroke by $30 \%$. In addition, total mortality was reduced by $21 \%$ and heart failure by $64 \%(25)$. In a 10 -year follow-up of the HUNT I study (1984-86), a consistent, positive association between systolic and diastolic blood pressure and mortality from stroke was found in men and women aged 70 years and older. This adds to the growing evidence that hypertension is a major risk factor for stroke mortality in the elderly (26).

\section{Cholesterol}

Hyperlipidaemia is a known risk factor for stroke, also in the elderly. An increasing net atherosclerotic pressure by $A p o B / A p o A 1$ is related to an increased risk of fatal stroke (27). Data from the HUNT studies show that the proportion of elderly with a total cholesterol level below $5 \mathrm{mmol} / \mathrm{L}$ has increased significantly between the survey in 1995-97 and 2006-08. Lowering of LDL cholesterol by $1 \mathrm{mmol} / \mathrm{L}$ in 1 year yields a relative risk reduction for stroke of 0.85 (CI 0.80-0.90), based on a meta-analysis of 39612 participants with a 5-year follow-up (28). A meta-analysis of 9 RCTs on secondary stroke prevention included elderly aged 6582 at inclusion and reported a $25 \%$ reduced stroke risk $(\mathrm{RR}=0.75$ (CI 0.53-0.85)) (29).

In the elderly, lower cholesterol levels are associated with increased mortality, probably due to malnutrition and underlying co-morbidities. It seems that increasing levels of total cholesterol is associated with an increase in HDL- cholesterol (30).

\section{Atrial fibrillation}

The prevalence of atrial fibrillation increases with age, and is associated with cardioembolic stroke, the most common stroke subtype in very old patients (31). The over-all prevalence in the population is estimated to $1 \%$, but reaches over $10 \%$ in age group 80 years and older $(32,33)$.

A study based on the Danish National Indicator Project found the prevalence of atrial fibrillation in stroke patients to be comparable in women and men, except for the oldest age groups, reporting a steeper increase for women after the age of 80 , and a decrease in prevalence in men over 85 (22).

Atrial fibrillation is more prevalent in the male population, but some studies have found a higher prevalence of atrial fibrillation in women at stroke onset. This seems to imply that women with atrial fibrillation have a higher risk of stroke, but it could also represent a so-called mortality displacement; a higher mortality at an earlier age for men with atrial fibrillation (22).

\section{Diabetes mellitus}

Diabetes is a well documented risk factor of stroke in the population $<75$ years. In the elderly the impact of diabetes on stroke risk is smaller. Intensive treatment of blood glucose in the elderly increases the risk of hypoglycaemia, which is more fateful due to the relative failure of compensatory mechanisms in the elderly. The 
metabolic syndrome predicts stroke, whereas impaired glucose intolerance alone is as strong a predictor as the metabolic syndrome itself (34).

\section{Smoking}

The importance of smoking as a risk factor decreases with years of survival in smokers. The smoking habits have changed considerably since the 1970s, especially in Northern Norway and Finland.

\section{DisCUSSION}

Increased survival, decreased mortality and a higher proportion of elderly in the population have resulted in a growing number of individuals with stroke.

In a study from Sweden the authors calculated that if the incidence rates were to stay constant, the absolute numbers of events would increase by $70 \%$ in men and $50 \%$ for women by the year 2050 (9). They further argue that the consequences of a growing population of elderly could be counterbalanced by a decline in incidence rates of $1-2 \%$ per year, based on the trend over the past 10 years in Sweden (9). This assumption is supported by similar analyses from Finland (11). A favourable incidence trend presupposes a continued high quality of health care and risk factor control in the population. In Finland the systematic effort since 1970 to lower the burden of risk factors for cardiovascular disease is rewarded with a reduction of stroke incidence to the average level of comparable Nordic countries (16).

Discrepancies between reported incidence rates among the elderly may be due to methodological challenges in monitoring incident strokes in this age group. The international standard for stroke incidence studies outlined by Sudlow and Warlow (1996) requires a population-based study with multiple overlapping case finding strategies (3). The national stroke database in Finland (PERFECT stroke) is hospital based, and does not meet these criteria, even though the hospitalization rate of stroke may be as high as $95-97 \%$ (35). To compare, the RIKS Stroke database, considered a model example of a national stroke registry, probably includes $80 \%$ of all stroke patients. In the very elderly the hospitalization rate tends to be lower, and the assessment and accuracy of diagnosis is more difficult due to comorbidity and restricted use of diagnostic procedures such as MRI. Wrong diagnosis of stroke among elderly and patients in nursing homes in particular, is common and partly due to so-called silent brain infarction with unspecific symptoms. Therefore, Ricci et al. recommended adding $30 \%$ events to estimate the true stroke rate among the oldest age group (36).

A recent Norwegian study reported a higher incidence of small vessel disease among elderly stroke patients than what is previously known. This is a small study, but points out an interesting area for future research; the pathophysiology of elderly stroke patients differs from that of the younger, and this may prove a challenge for both diagnostics, stroke prevention and stroke care (37).

According to the MONICA study (1982-1995) the reduced mortality in the age groups below 75 years in the Nordic countries can be attributed to increased stroke survival (17) due to better treatment and/or less severe stroke and it is likely that the same explanation applies to the very elderly.

There are few studies on prevalence relative to studies on incidence. As stroke is becoming more of a chronic illness, more studies on prevalence may be necessary in order to plan ahead for stroke patient care (6).

The impact of traditional risk factors may be different in the elderly compared to a younger population (31). Risk factors such as congestive heart failure, chronic renal disease and atrial fibrillation are significantly associated with stroke among those older than 85 years, whereas hypertension, hyperlipidaemia and heavy smoking are less relevant (38). Kammersgaard et al. having followed the very old in the Copenhagen Stroke Study found that pre-existing disability and age are far more important than traditional vascular risk factors (39). The level of dependency in daily living is a significant burden for the elderly stroke patients. Therefore, rehabilitation and task specific exercises aiming to regain a highest possible level of independency should have a priority among the elderly.

\section{CONCLUSION}

- The total stroke population is constantly getting older.

- The increasing prevalence of elderly individuals with stroke is one of the major challenges of future stroke care. There is a tendency to use less health care resources on the older stroke patients (15).

- The elderly stroke patients should be examined for unique risk factors and patterns which separate them from the younger stroke patients.

- Treatment and rehabilitation should focus on regaining independency and quality of life.

- The age-specific epidemiology of the elderly needs to be scrutinized further in larger studies in order to plan for future stroke care.

\section{REFERENCES}

1. Truelsen T, Bonita R, Jamrozik K. Surveillance of stroke: a global perspective. Int J Epidemiol $2001 ; 30$ (Suppl 1): S11-6.

2. Neurology in the elderly: more trials urgently needed. Lancet Neurol 2009; 8: 969. 
3. Sudlow CL, Warlow CP. Comparing stroke incidence worldwide: what makes studies comparable? Stroke 1996; 27: 550-8.

4. Feigin VL, Lawes CM, Bennett DA, et al. Worldwide stroke incidence and early case fatality reported in 56 population-based studies: a systematic review. Lancet Neurol 2009; 8: 355-69.

5. Ellekjaer H, Selmer R. [Stroke--similar incidence, better prognosis]. Tidsskr Nor Lageforen 2007; 127: 740-3.

6. Truelsen T, Piechowski-Jozwiak B, Bonita R, et al. Stroke incidence and prevalence in Europe: a review of available data. Eur J Neurol 2006; 13: 581-98.

7. Naess H, Waje-Andreassen U, Brogger J, et al. [Patients with acute cerebral infarction admitted to stroke unit]. Tidsskr Nor Lageforen 2011; 131: 814-8.

8. Meretoja A. PERFECT stroke: PERFormance Effectiveness, and Cost of treatment episodes in stroke. University of Helsinki, 2011.

9. Modig K, Drefahl S, Andersson T, et al. The aging population in Sweden: can declining incidence rates in MI, stroke and cancer counterbalance the future demographic challenges? Eur J Epidemiol 2012; 27: 139-45.

10. Hallstrom B, Jonsson AC, Nerbrand C, et al. Stroke incidence and survival in the beginning of the 21 st century in southern Sweden: comparisons with the late 20th century and projections into the future. Stroke 2008; 39: $10-5$

11. Sivenius J, Torppa J, Tuomilehto J, et al. Modelling the burden of stroke in Finland until 2030. Int J Stroke 2009; 4: 340-5.

12. Zhang Y, Chapman AM, Plested M, et al. The incidence, prevalence, and mortality of stroke in France, Germany, Italy, Spain, the UK, and the US: A literature review. Stroke Res Treat 2012; 2012: 436125.

13. Ellekjaer H. Epidemiological studies of stroke in a Norwegian population: incidence, risk factors and prognosis. Verdal, HUNT Research Center, Department of Community Medicine and General Practice, Faculty of Medicine, Norwegian University of Science and Technology (NTNU), 2000.

14. Appelros P, Nydevik I, Seiger A, et al. High incidence rates of stroke in Orebro, Sweden: Further support for regional incidence differences within Scandinavia. Cerebrovasc Dis 2002; 14: 161-8.

15. Russo T, Felzani G, Marini C. Stroke in the very old: a systematic review of studies on incidence, outcome, and resource use. J Aging Res 2011; 2011: 108785.

16. Vartiainen E, Jousilahti P, Alfthan G, et al. Cardiovascular risk factor changes in Finland, 1972-1997. Int J Epidemiol 2000; 29: 49-56.

17. http://statbank.ssb.no/statistikkbanken.

18. Sarti C, Stegmayr B, Tolonen H, et al. Are changes in mortality from stroke caused by changes in stroke event rates or case fatality? Results from the WHO MONICA Project. Stroke 2003; 34: 1833-40.

19. Zhu L, Fratiglioni L, Guo Z, et al. Association of stroke with dementia, cognitive impairment, and functional disability in the very old: a population-based study. Stroke 1998; 29: 2094-9.

20. Hornsten C, Molander L, Gustafson Y. The prevalence of stroke and the association between stroke and depression among a very old population. Arch Gerontol Geriatr 2012; 55: 555-9.

21. Rastas S, Verkkoniemi A, Polvikoski T, et al. Atrial fibrillation, stroke, and cognition: a longitudinal population-based study of people aged 85 and older. Stroke 2007 ; 38: 1454-60.

22. Andersen KK, Andersen ZJ, Olsen TS. Age- and gender-specific prevalence of cardiovascular risk factors in 40,102 patients with first-ever ischemic stroke: a Nationwide Danish Study. Stroke 2010; 41: 2768-74.

23. Rojas JI, Zurru MC, Romano M, et al. Acute ischemic stroke and transient ischemic attack in the very old risk factor profile and stroke subtype between patients older than 80 years and patients aged less than 80 years. Eur J Neurol 2007; 14: 895-9.

24. Beckett NS, Peters R, Fletcher AE, et al. Treatment of hypertension in patients 80 years of age or older. $N$ Engl J Med 2008; 358: 1887-98.

25. Bulpitt CJ, Beckett NS, Peters R, et al. Blood pressure control in the Hypertension in the Very Elderly Trial (HYVET). J Hum Hypertens 2012; 26: 157-63.

26. Ellekjaer H, Holmen J, Vatten L. Blood pressure, smoking and body mass in relation to mortality from stroke and coronary heart disease in the elderly. A 10-year follow-up in Norway. Blood Pressure 2001; 10: 156-63.

27. Walldius G, Aastveit AH, Jungner I. Stroke mortality and the apoB/apoA-I ratio: results of the AMORIS prospective study. J Intern Med 2006; 259: 259-66.

28. Baigent C, Blackwell L, Emberson J, et al. Efficacy and safety of more intensive lowering of LDL cholesterol: a meta-analysis of data from 170,000 participants in 26 randomised trials. Lancet 2010; 376: 1670-81.

29. Afilalo J, Duque G, Steele R, et al. Statins for secondary prevention in elderly patients: a hierarchical Bayesian meta-analysis. J Am Coll Cardiol 2008; 51: 37-45.

30. Upmeier E, Lavonius S, Heinonen $\mathrm{P}$, et al. Longitudinal changes in serum lipids in older people the Turku elderly study 1991-2006. Age Ageing 2011; 40: 280-3.

31. Chen RL, Balami JS, Esiri MM, et al. Ischemic stroke in the elderly: an overview of evidence. Nature Rev Neurol 2010; 6: 256-65. 
32. Feinberg WM, Blackshear JL, Laupacis A, et al. Prevalence, age distribution, and gender of patients with atrial fibrillation. Analysis and implications. Arch Intern Med 1995; 155: 469-73.

33. Go AS, Hylek EM, Phillips KA, et al. Prevalence of diagnosed atrial fibrillation in adults: national implications for rhythm management and stroke prevention: the AnTicoagulation and Risk Factors in Atrial Fibrillation (ATRIA) Study. JAMA 2001; 285: 2370-5.

34. Wang J, Ruotsalainen S, Moilanen L, et al. The metabolic syndrome predicts incident stroke: a 14-year follow-up study in elderly people in Finland. Stroke 2008; 39: 1078-83.

35. Mahonen M, Salomaa V, Keskimaki I, et al. The feasibility of combining data from routine Hospital Discharge and Causes-of-Death Registers for epidemiological studies on stroke. Eur J Epidemiol 2000; 16: 815-7.

36. Ricci S, Celani MG, La Rosa F, et al. SEPIVAC: a community-based study of stroke incidence in Umbria, Italy. J Neurol Neurosurg Psychiatry 1991; 54: 695-8.

37. Ihle-Hansen H, Thommessen B, Wyller TB, et al. Risk factors for and incidence of subtypes of ischemic stroke. Funct Neurol 2012; 27: 35-40.

38. Arboix A, Miguel M, Ciscar E, et al. Cardiovascular risk factors in patients aged 85 or older with ischemic stroke. Clin Neurol Neurosurg 2006; 108: 638-43.

39. Kammersgaard LP, Jorgensen HS, Reith J, et al. Short- and long-term prognosis for very old stroke patients. The Copenhagen Stroke Study. Age Ageing 2004; 33: 149-54. 